\title{
Progression of muscle histopathology but not of spliceopathy in myotonic dystrophy type 2
}

\author{
Rosanna Cardani ${ }^{\mathrm{a}}$, Marzia Giagnacovo ${ }^{\mathrm{b}}$, Giulia Rossi ${ }^{\mathrm{c}}$, Laura V. Renna ${ }^{\mathrm{d}}$, \\ Enrico Bugiardini ${ }^{\mathrm{e}}$, Chiara Pizzamiglio ${ }^{\mathrm{e}}$, Annalisa Botta ${ }^{\mathrm{c}}$, Giovanni Meola ${ }^{\mathrm{a}, \mathrm{e}, *}$ \\ ${ }^{a}$ Laboratory of Muscle Histopathology and Molecular Biology, IRCCS Policlinico San Donato, Milan, Italy \\ ${ }^{\mathrm{b}}$ Department of Biology and Biotechnologies, University of Pavia, Pavia, Italy \\ ${ }^{\mathrm{c}}$ Department of Biomedicine and Prevention, Tor Vergata University of Rome, Rome, Italy \\ ${ }^{\mathrm{d}}$ Department of Biosciences, University of Milan, Milan, Italy \\ ${ }^{\mathrm{e}}$ Department of Neurology, University of Milan, IRCCS-Policlinico San Donato, Milan, Italy
}

Received 2 April 2014; received in revised form 7 June 2014; accepted 17 June 2014

\begin{abstract}
Myotonic dystrophy type 2 (DM2) is an autosomal dominant progressive disease involving skeletal and cardiac muscle and brain. It is caused by a tetranucleotide repeat within the first intron of the $C N B P$ gene that leads to an alteration of the alternative splicing of several genes. To understand the molecular mechanisms that play a role in DM2 progression, the evolution of skeletal muscle histopathology and biomolecular findings in successive biopsies have been studied. Biceps brachii biopsies from 5 DM2 patients who underwent two successive biopsies at different years of age have been used. Muscle histopathology has been assessed on sections immunostained with fast or slow myosin. FISH in combination with MBNL1-immunofluorescence has been performed to evaluate ribonuclear inclusion and MBNL1 foci dimensions in myonuclei. Gene and protein expression and alteration of alternative splicing of several genes have been evaluated over time. All DM2 patients examined show a worsening of muscle histopathology and an increase of foci dimensions over time. The progressive worsening of myotonia in DM2 patients may be due to the decrease of CLCN1 mRNA observed in all patients examined. However, a worsening of alternative splicing alterations has not been evidenced over time. The data obtained in this study confirm that DM2 is a slow progression disease since histological and biomolecular alterations observed in skeletal muscle are minimal even after 10-year interval. The data indicate that muscle morphological alterations evolve more rapidly over time than the molecular changes thus indicating that muscle biopsy is a more sensitive tool than biomolecular markers to assess disease progression at muscle level.
\end{abstract}

(C) 2014 Elsevier B.V. All rights reserved.

Keywords: Myotonic dystrophy type 2; Ribonuclear inclusions; Disease progression; Muscle histology; Alternative splicing

\section{Introduction}

Myotonic dystrophy type 2 (DM2) is characterized by autosomal dominant progressive myopathy, myotonia

\footnotetext{
* Corresponding author at: Department of Neurology, University of Milan, IRCCS Policlinico San Donato, Piazza E. Malan 1, 20097 San Donato Mil., Milan, Italy. Tel.: +3902 52774480; fax: +39 025274717. E-mail address: giovanni.meola@unimi.it (G. Meola).
}

and multiorgan involvement [1-3]. DM2 mutation consists in the expansion of an unstable CCTG tetranucleotide within the first intron of the CCHC-type zinc finger, nucleic acid-binding protein $(C N B P)$ gene [4] on chromosome $3 \mathrm{q} 21$ and is thus similar to the untranslated (CTG)n repeat in the DMPK gene causing DM type 1 (DM1) [5,6]. The prevailing paradigm is that both disorders are toxic RNA diseases since 
CUG/CCUG-containing mutant transcripts aggregate in cell nuclei as ribonuclear inclusions that alter the activity of specific RNA-binding proteins such as MBNL1 and CUGBP1 [7-9]. This results in a "spliceopathy", i.e. alteration of alternative splicing of several downstream effector genes, which is thought to account, at least in part, for multiorgan involvement [10,11]. At skeletal muscle level, DM2 has been defined as "a disease of type 2 fibers" since these myofibers are selectively affected by atrophy and central nucleation [12,13]. The definitive diagnosis of DM2 can be achieved through the detection of the DM2 mutation either at DNA or RNA level with the identification of the expanded RNAs as ribonuclear inclusions by in situ hybridization [14-16]. The progression of the DM2 is slow and prognosis and life expectancy are more favorable than in DM1. Life expectancy may be reduced only in single DM2 cases, mainly if there is severe cardiac involvement with malignant rhythm abnormalities. Thus DM2 is considered a clinically milder disease than DM1. While DM2 disease progression is well documented by clinical reports mainly on cardiac, central nervous system and neuromuscular involvement [17,18], no literature data are available on correlations between muscle involvement progression with biomarkers for disease severity. Therefore in this work we have studied the progression of the muscular involvement in relation to the evolution of skeletal muscle histopathology and biomolecular findings to better prognosticate patients with DM2. Despite the limited number of patients examined, this study may contribute to better understand the molecular mechanisms underlying DM2 pathology.

\section{Materials and methods}

This study was authorized by the Institutional Ethics Committee (ASL MI2-Melegnano via VIII Giugno, Milan) and was conducted according to the principles expressed in the Declaration of Helsinki, the institutional regulation and Italian laws and guidelines. All blood samples and muscle biopsies were used for this study after receiving written informed consent from the patients.

\subsection{Patients}

Human muscle biopsies were taken under sterile conditions from 5 DM2 patients who underwent two successive biceps brachii biopsies from the same side at different years of age. Biceps brachii biopsies from two patients with classical DM1 phenotype and from two healthy subjects were also used. The diagnosis of DM was based upon the clinical diagnostic criteria set by the International Consortium for Myotonic Dystrophy [19]. Muscle strength was measured using the modified 5-point MRC scale (Medical Research Council) in the upper and lower limbs for a total of 150 maximum score. Basal
EKG and echocardiographic evaluation was performed in all patients.

\subsection{Molecular analysis of the CCTG expansion in the CNBP gene}

Genomic DNA has been extracted from muscle homogenates with a salting-out procedure [20]. Detection of the CCTG expansion has been obtained with a Long-PCR based method as previously described [16].

\subsection{Muscle histopathology}

Muscle tissue was fresh-frozen in isopentane cooled in liquid nitrogen. Histopathological analyses were performed on serial sections $(8 \mu \mathrm{m})$ processed for routine histological or histochemical stainings. A standard myofibrillar ATPase staining protocol was used after preincubation at $\mathrm{pH} 4.3,4.6$, and 10.4 [21]. The most typical alterations, such as nuclear clump fibers (i.e. aggregates of myonuclei with a thin rim of cytoplasm), nuclear centralization and fiber size variability were evaluated on serial muscle sections.

\subsection{Immunohistochemistry}

Serial transverse muscle cryostat sections $6 \mu \mathrm{m}$ thick were cut for immunohistochemical staining (IHC). Sections were air-dried and rehydrated in phosphate buffer $\mathrm{pH} 7.4$ (PBS). Non-specific binding sites were blocked with normal goat serum (NGS; Dako, Denmark) at a dilution 1:20 in PBS containing $2 \%$ bovine serum albumin (BSA; Sigma-Aldrich, St. Louis, MO) for $20 \mathrm{~min}$ at room temperature (RT). Mouse monoclonal primary antibodies against two different myosin heavy chain (MHC) isotypes were used at the following dilutions: MHfast, 1:400 in PBS $+2 \%$ BSA; MHCslow, 1:400 in PBS $+2 \%$ BSA (Sigma-Aldrich, St. Louis, MO). Each antibody was applied for $1 \mathrm{~h}$ at RT. After washing in PBS 3 times for $5 \mathrm{~min}$, sections were incubated with goat anti-mouse biotinylated secondary antibody diluted $1: 300$ in $\mathrm{PBS}+2 \%$ BSA for $1 \mathrm{~h}$ at RT. After PBS washing $(3 \times 5 \mathrm{~min})$, sections were incubated with Vectastain Elite ABC kit (Vector Laboratories; Burlingame, CA) for $30 \mathrm{~min}$ and then exposed to the 3,3'diaminobenzidine tetrahydrochloride (DAB; SigmaAldrich, St. Louis, MO) chromogen reaction solution for $10 \mathrm{~min}$. Nuclei were counterstained with Mayer's haematoxylin. Quantitative evaluation of fiber diameter was made as described previously by Vihola et al. [12] on images with Image $\mathbf{J}$ (Scion Corporation, Frederick, MD) on images taken with a light microscope $(160 \times$, original magnification). The size of muscle fibers was assessed by measuring the "smallest fiber diameter". All data were elaborated using Microcal Origin (Microcal Software Inc., Northampton, MA, USA). The metahistograms were normalized to normal mean diameter for men and 
women. Atrophy and hypertrophy factors were also calculated [21].

\subsection{Fluorescence in situ hybridization (FISH) combined with MBNL1 immunofluorescence}

FISH was performed on DM1 and DM2 muscle frozen sections using a $(\mathrm{CAGG})_{5}$ or $(\mathrm{CAG})_{6}-\mathrm{CA}$ probe (IDT, Coralville, IA, USA) as previously reported by Cardani et al. [14] to verify the presence of ribonuclear inclusions. In brief, $6 \mu \mathrm{m}$ thick transverse cryostatic sections were air dried for $30 \mathrm{~min}$ and fixed with $2 \%$ paraformaldehyde for $30 \mathrm{~min}$ at $4{ }^{\circ} \mathrm{C}$. The sections were then washed in PBS and permeabilized for $5 \mathrm{~min}$ in $2 \%$ acetone in PBS, prechilled at $-20{ }^{\circ} \mathrm{C}$. After washing in PBS, sections were incubated in $40 \%$ formamide and $2 \times$ saline solution citrate (SSC) for $10 \mathrm{~min}$ at RT, and hybridized for $2 \mathrm{~h}$ at $37^{\circ} \mathrm{C}$, with $1 \mathrm{ng} / \mu \mathrm{L}$ probe $(\mathrm{CAGG})_{5}$ or $(\mathrm{CAG})_{6}-\mathrm{CA}$ Texas red labeled probes in $30 \%$ formamide, $2 \times \mathrm{SSC}$, $0.02 \%$ BSA, $67 \mathrm{ng} / \mu \mathrm{L}$ yeast tRNA, $2 \mathrm{mM}$ vanadyl ribonuclease complex (all these reagents were from Sigma-Aldrich, St. Louis, MO). The sections were washed first in $40 \%$ formamide and $2 \times \mathrm{SSC}$ at $45^{\circ} \mathrm{C}$ for $30 \mathrm{~min}$, then in $1 \times \mathrm{SSC}$ at $45^{\circ} \mathrm{C}$ for $15 \mathrm{~min}$ and another $1 \times$ SSC wash at RT. The FISH-labeled sections were then processed for the immunofluorescence detection of MBNL1, as follows. The sections were pre-incubated for 20 min at RT with 5\% NGS in PBS containing $2 \%$ BSA and then incubated overnight at $4{ }^{\circ} \mathrm{C}$ with a rabbit polyclonal antibody recognizing MBNL1 (kind gift of Prof. C.A. Thornton, University of Rochester, New York, USA); the primary antibody was diluted 1:1000 in PBS containing $2 \%$ BSA. The sections were washed in PBS and then incubated for $1 \mathrm{~h}$ at RT with a goat anti-rabbit Alexa488-labeled antibody (Molecular Probes, Life Technologies, Milan, Italy), diluted 1:200 in PBS, counterstained for DNA with DAPI $(1 \mu \mathrm{g} / \mathrm{ml}$; Sigma-Aldrich, St. Louis, MO), and mounted with Mowiol (Calbiochem, Milan, Italy). As controls, some slides were processed as described above but omitting the incubation with the primary antibody.

\subsection{Morphometric evaluation of the ribonuclear inclusions and MBNL1 foci}

RI and MBNL1 foci dimensions have been measured in patients \#1, \#2, \#3 and \#5. Double-labeled sections by FISH and MBNL1 were analyzed by confocal microscopy, using a Leica TCS SP2 AOBS system: for fluorescence excitation, an $\mathrm{Ar} / \mathrm{Vis}$ laser at $488 \mathrm{~nm}$ for Alexa488, and $\mathrm{He} / \mathrm{Ne}$ laser at 543 for Texas red; the laser intensity, pinhole opening, signal amplification and image spacing along the $z$ axis were kept constant, and images were recorded in the $1024 \times 1024$ pixels format, using a $63 \times$ oil immersion objective. For each section, 60 ribonuclear inclusions and the corresponding MBNL1-containing foci were measured. Acquisitions were carried out at the Centro
Interdipartimentale di Microscopia Avanzata (CIMA) of the University of Milan, Italy. The size and fluorescence intensity of the ribonuclear inclusions and MBNL1 foci were measured using the Leica Confocal Software, and the mean values and standard deviations were calculated.

\subsection{Western blot analysis}

Whole cell extracts were obtained from fifteen-twenty consecutive muscle cryostat sections $10 \mu \mathrm{m}$ thick homogenized in $60 \mu \mathrm{l}$ of $50 \mathrm{mM}$ TrisHCl with $5 \%$ SDS (pH 7.5). After incubating on ice for $15 \mathrm{~min}$, samples were centrifuged at $5700 \mathrm{~g}$ for $12 \mathrm{~min}$ at $4^{\circ} \mathrm{C}$, and supernatant was collected and stored at $-80^{\circ} \mathrm{C}$. Pellets were resuspended in $50 \mathrm{mM}$ Tris $\mathrm{HCl}$ with $5 \%$ SDS $(\mathrm{pH}$ 7.5) and stored at $-80^{\circ} \mathrm{C}$. Protein concentration in each sample was determined by using BCA Protein Assay (Bio-Rad Laboratories, Milano, Italy). An equal amount of protein was loaded per lane and electrophoresed on $12 \%$ sodium dodecyl sulfate-polyacrylamide gels, and then transferred to nitrocellulose membranes (Bio-Rad Laboratories, Milano, Italy). After blocking non specific sites in TrisHCl buffer pH 7.5 (TBS) containing 5\% BSA for $30 \mathrm{~min}$ at $42{ }^{\circ} \mathrm{C}$, membranes were incubated overnight at $4{ }^{\circ} \mathrm{C}$ with mouse monoclonal anti CUGBP1 (clone 3B; 1:1000; SantaCruz Biotechnology, Heidelberg, Germany) or with rabbit polyclonal anti-ZNF9/CNBP (1:1000) [22]. After several washes in TBS $+0.2 \%$ Tween 20 or TBS $+0.3 \%$ Tween 20 respectively, membranes were incubated with horseradish peroxidase-conjugated (Jackson ImmunoResearch Laboratories, Baltimore, PA) goat anti-mouse $(1: 5000$ in $\mathrm{TBS}+5 \%$ BSA $+0.2 \%$ Tween20) or goat anti-rabbit $(1: 10,000$ in TBS $+5 \%$ BSA $+0.2 \%$ Tween 20) secondary antibodies. Membranes were washed and immune complexes were detected using the ECL detection system (Amersham Pharmacia Biotech, Piscataway, NJ). GAPDH (polyclonal antibody diluted 1:80,000; Sigma-Aldrich, St. Louis, MO) was used as internal control to verify and correct for loading error. Blots have been performed in triplicate.

\subsection{Study of alternative splicing}

Frozen muscle samples were practiced for the extraction of total RNA using TRIzol reagent (Gibco BRL, Gaithersburg, MD) and $1 \mu \mathrm{g}$ of RNA was reverse transcribed according to the cDNA protocol of the High Capacity cDNA Archive kit (Applied Biosystems, Foster City, CA). Splicing pattern profile of the $I R, C L C N 1$, $M B N L 1$ and SERCA1 genes was carried out as described [23-25]. Total PCR products, obtained within the linear range of amplification, were electrophoresed on $2.5 \%$ agarose gel. Quantitative analysis of the amplified products was performed using SybrGreenII-stained gels (Perkin-Elmer Life Science, Massachusetts, USA) scanned on a fluorimager 595 (Amersham Biosciences, Buckinghamshire, UK). The intensity of each band and 
the fraction of abnormally (or pathologically) spliced (AS) isoforms (AS-isoforms/total) were quantified by densitometry using ImageQuant software. Control of the RT-PCR reaction was based on the expression level of the glucose phosphate isomerase housekeeping gene (GPI) and all amplifications have been carried out in triplicate using independent cDNA samples.

\subsection{QRT-PCR expression analysis of the CLCN1 and $C N B P$ genes}

Following RNA extraction and retro-transcription, cDNAs of DM2 samples were also used to quantify the expression level of the $C L C N 1$ and $C N B P$ genes. The total expression of mentioned genes was evaluated using specific TaqMan gene expression assays: CLCN1 [Hs00163961_ml] and $C N B P$ [Hs00231535_ml] (Applied Biosystems, Foster City, CA). The VIC-labeled $\beta_{2}$-microglobulin gene (B2M: GenBank accession \#NM_004048) was used as housekeeping internal control gene, as described [26]. The simultaneous measurement of genes-FAM/B2M-VIC expression allow to normalize the amount of cDNA added per sample. Each PCR reaction was performed in triplicate using the TaqMan Universal PCR Master Mix and the ABI PRISM 7500 Fast System (Applied Biosystems, Foster City, CA). A comparative threshold cycle (Ct) was used to determine gene expression compared to a calibrator (median value of control subjects). Hence, steady-state mRNA levels were expressed in $n$-fold difference relative to the calibrator. For each sample, genes' $\mathrm{Ct}$ value was normalized using the formula $\Delta \mathrm{Ct}=\mathrm{Ct}$ genes $-\mathrm{Ct} \mathrm{B} 2 \mathrm{M}$. To determine the relative expression levels, the following formula was used: $\Delta \Delta \mathrm{Ct}=\Delta \mathrm{Ct}$ sample $-\Delta \mathrm{Ct}$ calibrator. The value adopted to plot relative gene expression was calculated using the expression $2^{-\Delta \Delta \mathrm{Ct}}$

\subsection{Statistical analysis}

The Student's $t$-test or the non parametric Mann-Whitney test has been performed to determine significant differences between the two successive muscle biopsies in each patient.

\section{Results}

\subsection{Patients}

Clinical data on DM2 patients used in this study are reported in Table 1. Patient \#1 was evaluated at the age of 35. She presented a severe weakness conditioning gait difficulty. Both myotonia and myalgia were reported. At the second evaluation, 10 years later, a further deterioration of muscle strength and a mild decrease of myotonia were evidenced. At the first examination patient \#2 (33 yr old) reported stiffness and myalgia since he was 19 years old. Neurological examination showed normal strength and trophism. Myotonia with warm up phenomenon was evidenced. Five years later, when the second biopsy was performed, the clinical phenotype was unchanged and the patient showed a clinical improvement of myotonia and myalgia due to mexiletine treatment. Cardiac parameters were stationary. Patient \#3 started to complain of proximal leg weakness at the age of 36 and he was evaluated for the first time at the age of 54 . He presented widespread myalgia, mild hand percussion myotonia and proximal weakness both to the upper and lower limbs. During the second evaluation, 4 years later, he reported an improvement of myotonia and myalgia due to mexiletine and a worsening of proximal weakness. Cardiac ECG showed the appearance of a BAV I grade block (PR $>200 \mathrm{~ms}$ ). Patient \#4 was visited twice, first at 58 and then at 60 years of age. The clinical findings were comparable and were characterized by proximal weakness, moderate hand myotonia and myalgia. Finally, patient \#5 complained muscle pain since he was 30 years old. The first biopsy was performed at the age of 51. At that time he presented grip myotonia, myalgia and proximal weakness. He was admitted to our department for a second biopsy 2 years later with an unaltered clinical situation.

Table 1

Clinical data on DM2 patients used in this study.

\begin{tabular}{|c|c|c|c|c|c|}
\hline $\mathrm{Pt}$ & $\# 1$ & $\# 2$ & \#3 & $\# 4$ & $\# 5$ \\
\hline Time between evaluation & 10 & 5 & 4 & 2 & 2 \\
\hline Age baseline & 35 & 33 & 54 & 58 & 51 \\
\hline Age follow-up & 45 & 38 & 58 & 60 & 53 \\
\hline MRC muscle biopsied follow-up & 2 & 5 & 4 & 5 & 4 \\
\hline MRC total baseline & 93 & 150 & 139.3 & 145 & 143 \\
\hline MRC total Follow-up & 88 & 150 & 136 & 145 & 143 \\
\hline QRS baseline & 85 & 104 & 86 & 104 & 116 \\
\hline QRS follow-up & 92 & 100 & 106 & 106 & 116 \\
\hline $\mathrm{FE} \%$ baseline & 63.5 & 76.5 & 62.2 & n.d. & 65.8 \\
\hline $\mathrm{FE} \%$ follow-up & 63 & 70 & 66.7 & 56.4 & 61.4 \\
\hline
\end{tabular}

${ }^{\mathrm{a}}$ Medical Research Council, scale for muscle strength; scale (0-5 grade) on 15 muscles at both side in the upper and lower limbs for a total of 150 maximum score. 


\subsection{Muscle histopathology}

All DM2 patients examined show a worsening of muscle histopathology over time (Figs. 1 and 2). Central nucleation is always present involving prevalently type 2 fibers (Figs. 1A-C) and the percentage of type 2 fibers presenting central nucleation increases in the second biopsy compared to the first one (Fig. 1C). The majority of nuclear clumps fibers present in the muscle sections examined, express only the MHC fast myosin, while nuclear clumps fibers expressing only MHC slow myosin are rarely or no present (Fig. 1A and B). The number of MHCfast positive nuclear clumps appears to be higher in the second biopsy than in the first one in all patients (Fig. 1D). The metahistograms based on the analysis of fiber diameters on immunostained muscle sections and the evaluation of atrophy (AF) and hypertrophy (HF) factors are reported in Fig. 2. Analysis of MHC fast and slow myosin immunostained muscle sections allows us to detect and measure fibers smaller than $5 \mu \mathrm{m}$, including all nuclear clump fibers which are recognizable by the presence of a thin rim of immunoreaction around the nuclei. An increase of both type 1 and type 2 fiber AF is evident between the first and the second biopsy in all patients examined. However, as expected in DM2 muscle, atrophy is more marked in type 2 than type 1 fibers, except for one patient $(\mathrm{Pt} \# 2)$ presenting a very mild phenotype, where type 1 and type 2 fiber atrophy appears to be similar in both biopsies (Fig. 2).

\subsection{Ribonuclear inclusions (RI) and MBNL1 foci dimensions}

RI and MBNL1 foci dimensions have been measured on images obtained at confocal microscopy of the two successive DM2 muscle biopsies to verify if an increase of foci dimensions is evident over time. As expected, a clear co-localization of ribonuclear inclusions and MBNL1 foci is evident in all the muscle biopsies examined. In all DM2 patients considered, areas of RI or MBNL1 foci (Fig. 3) and the corresponding fluorescence intensities (data not shown) show a significant increase of both foci type dimensions in the second biopsy compared to those observed in the first one. However the fold increase of foci dimensions does not appear to correlate with the time elapsed between the two successive biopsies. Interestingly, the RI and MBNL1 foci in DM2 muscle appear to be significantly larger than those observed in DM1 muscle nuclei (Fig. 3E-I).

\subsection{Western blotting analysis}

The protein levels of CUGBP1 and CNBP have been evaluated in the two successive muscle biopsies from DM2 patients by western blotting analysis. CUGBP1 protein level is higher in the second biopsies in all the patients examined (Fig. 4A and B). The most evident increase of CUGBP1 expression between the first and the second biopsy (4.7-fold increase) is observable in patient
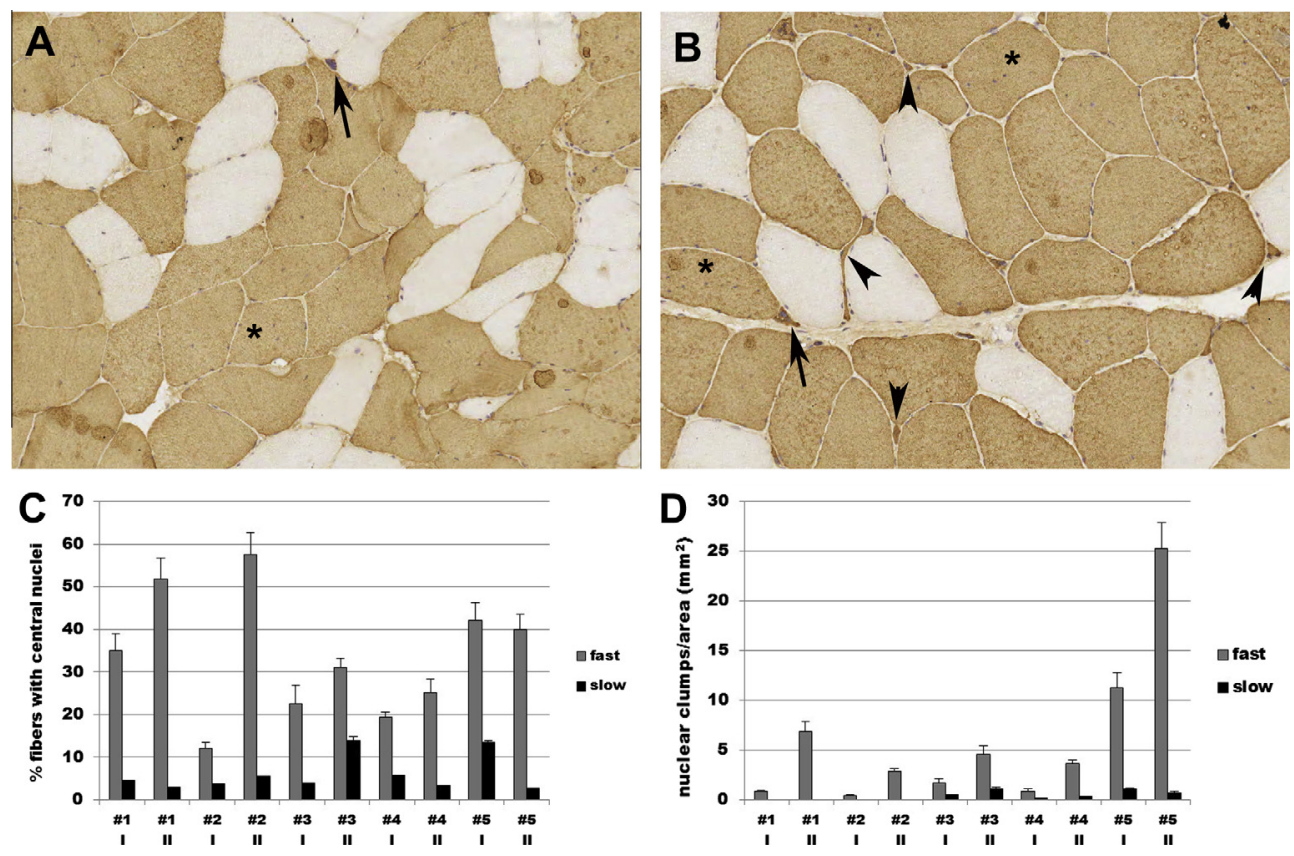

Fig. 1. Fast myosin immunostaining of skeletal muscle transverse sections obtained from the first biopsy (A) and from the second biopsy (B) of patient \#4. Type 2 fibers (fast positive fibers) are stained in brown. Note the increase of the number of very atrophic fibers fast positive (arrowheads) and of type 2 fiber with central nuclei (asterisks). Arrows indicate type 2 nuclear clumps. Original magnification $400 \times$. The percentage of fibers with central nuclei (C) and the number of nuclear clumps per area (D). Histograms represent the mean $\pm \mathrm{SD}$ of quantifications made on three different sections for each biopsy. 

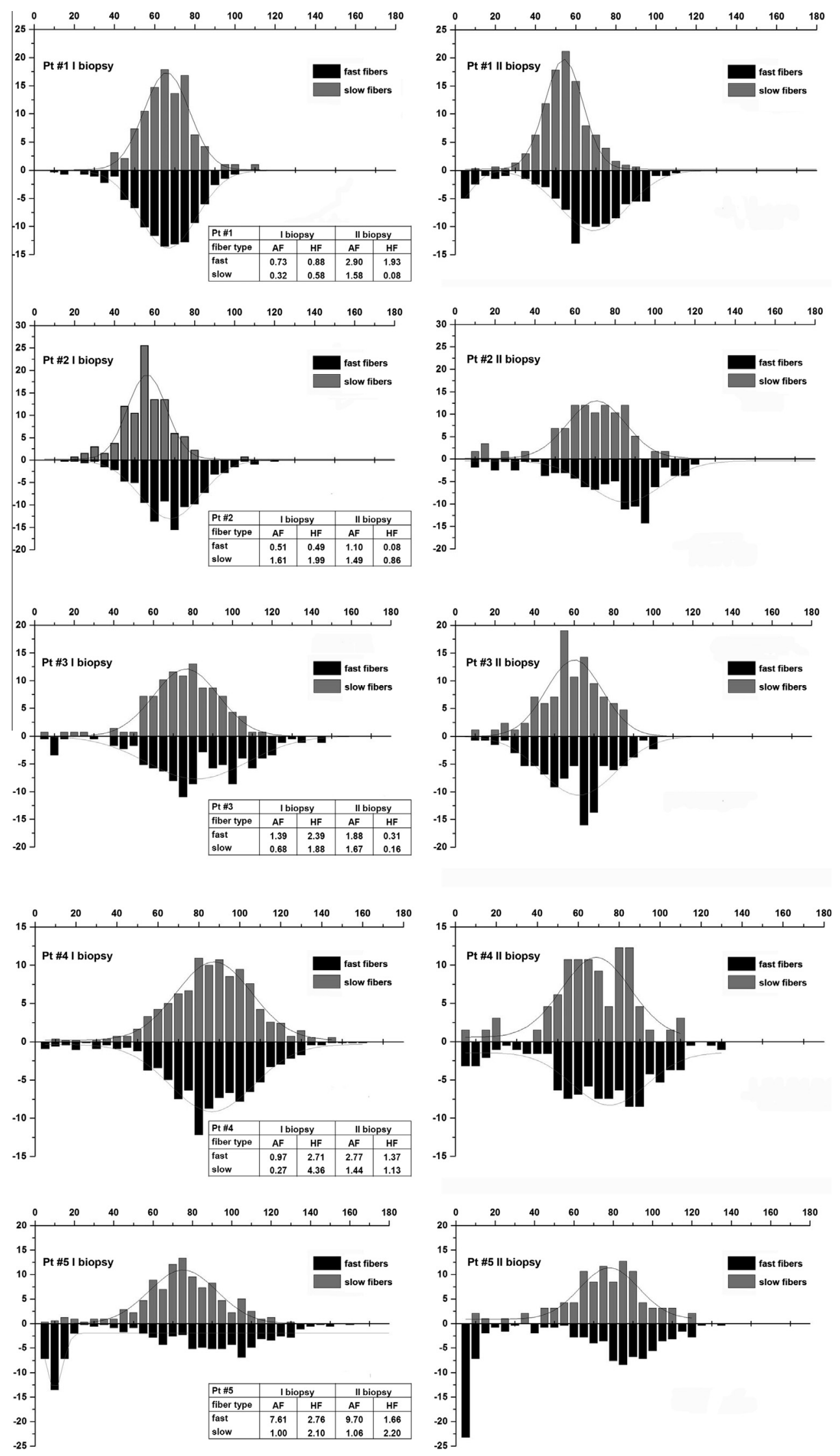

Fig. 2. Metahistograms obtained from the analysis of muscle fiber diameters in the first (left) and second (right) muscle biopsy from 5 DM2 patients. The results are based on sections immunostained for MHC fast or slow myosin. Tables show the relative atrophy or hypertrophy factors in both biopsies of each patient. 

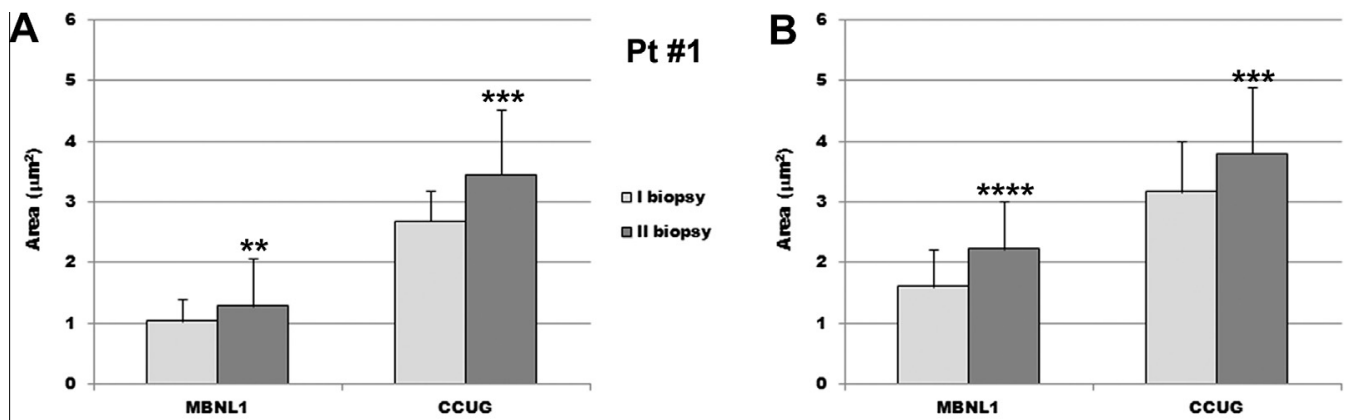

Pt \#2

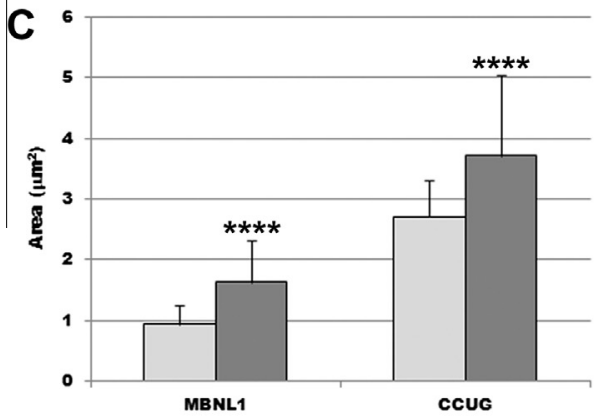

\section{Pt \#3}
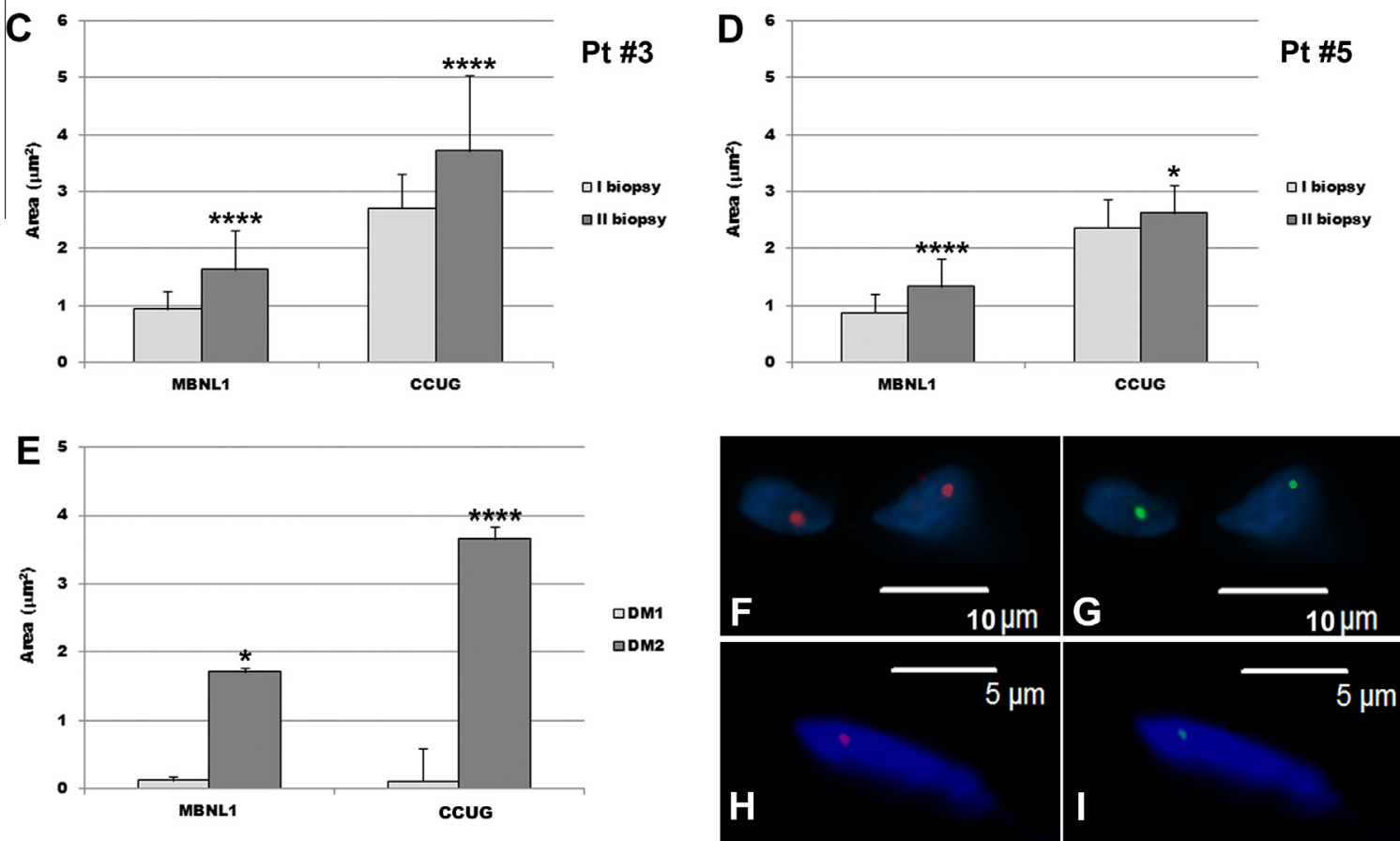

Fig. 3. Ribonuclear inclusions and MBNL1 foci dimensions in patients \#1 (A), \#2 (B), \#3 (C) and \#5 (D). Histograms indicate the mean \pm SD of area of ribonuclear inclusions and MBNL1 nuclear foci obtained at confocal laser scanning microscopy in the first and second biopsy. Comparison between foci dimensions in 2 DM1 patients and those observed in 2 DM2 patient (\#1 and \#3, second biopsy) (E). FISH in combination with MBNL1 immunofluorescence performed on DM2 muscle (F and G) and on DM1 muscle ( $\mathrm{H}$ and $\mathrm{I}$ ) shows that ribonuclear inclusions (F and H; red spots) and MBNL1 foci ( $\mathrm{G}$ and I; green spots) colocalize in nuclei (blue, DAPI) and appear to be larger in DM2 than in DM1. Mann-Whitney test: ${ }^{*} p<0.05$; ${ }^{* *} p<0.01 ;{ }^{* * *} p<0.001 ;{ }^{* * * *} p<0.0001$.

\#1 whose second biopsy was performed after 10 years from the first one (Fig. 4B). CUGBP1 expression in DM2 muscle biopsies appears to be similar or lower than those observed in control biopsies (Fig. 4A).

CNBP protein levels in DM2 muscles examined are similar in the two successive biopsies and appear to be reduced compared with control samples (Fig. 4C and D).

\subsection{Alternative splicing}

In this work we have analyzed splicing isoforms of $I R$, $C L C N 1, S E R C A 1$ and $M B N L 1$ genes in two successive muscle biopsies from DM2 patients to understand if a worsening of clinical phenotype is associated to a worsening of alternative splicing alteration of these genes in DM2 skeletal muscle. As expected, a statistically significant alteration of alternative splicing of the genes examined in this work is present in all the DM2 muscle biopsies considered as compared to controls (Fig. 5). However, no difference or a slight increase in the expression of $I R$ and $M B N L 1$ pathological isoforms is observable between the first and the second biopsy of all the patients examined (Fig. 5). SERCA1 and CLCN1 genes splicing pattern is however more variable and appears worsened in patients \#3, \#4 and \#5 with no obvious correlation with the muscle phenotypes (Fig. 5). No correlation is evident between the variation of spliceopathy and the time elapsed between the two successive biopsies.

\subsection{CLCN1 and CNBP $m R N A$ expression level}

CLCN1 mRNA expression level shows a decrease in the second biopsy of all the patients examined. However this reduction appears to be statistically significant only for patient \#1 whose second biopsy was performed 10 years 

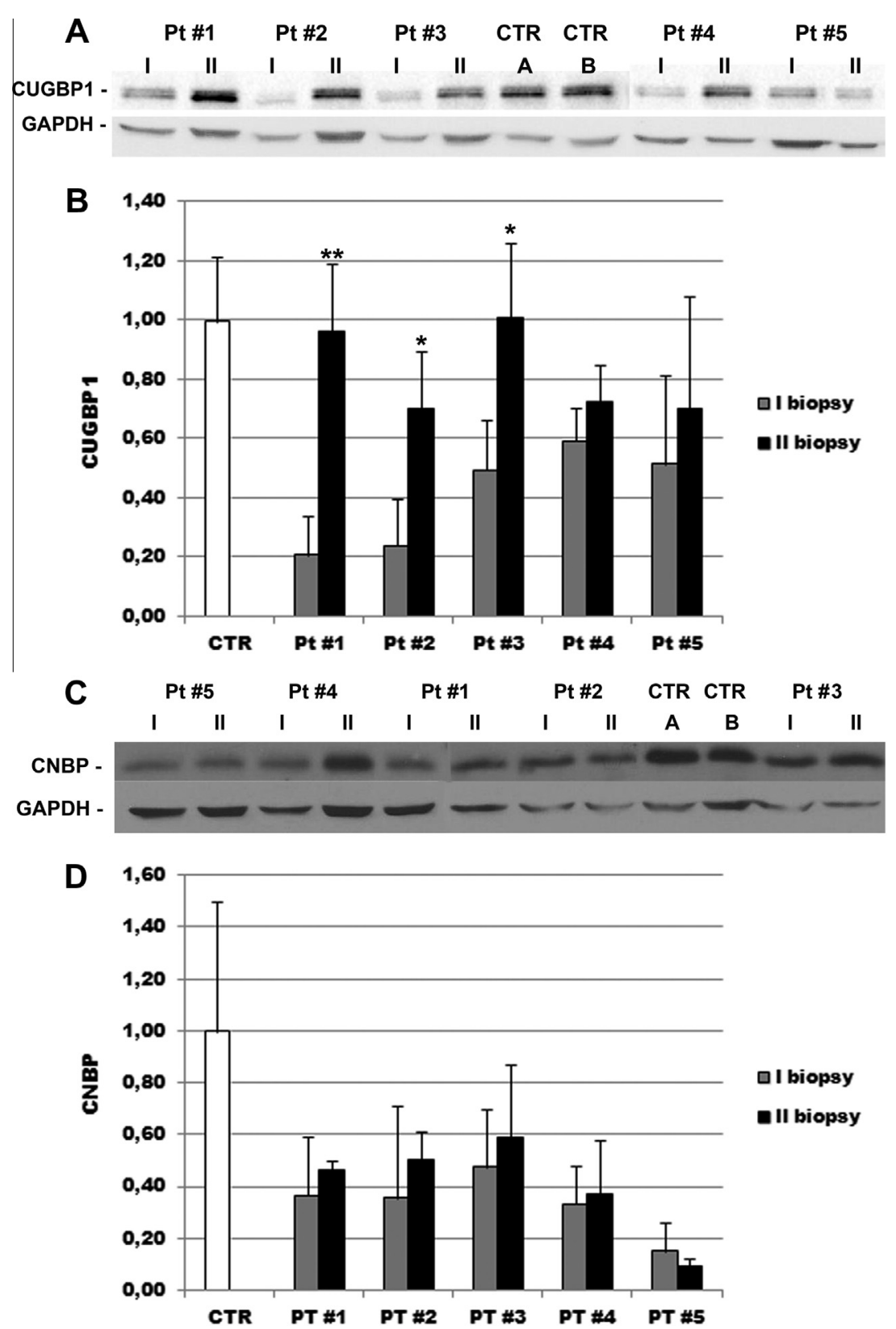

Fig. 4. Western blot analysis of CUGBP1 and CNBP protein expression in muscle samples from 2 healthy subjects and from two successive biopsies of 5 DM2 patients. Density of the bands has been normalized with GAPDH expression used as internal control (A and C). Histograms represent mean values $\pm \mathrm{SD}$ of CUGBP1 and CNBP protein expression analyzed by densitometry (B and D). Student's $t$-test: ${ }^{*} p<0.05 ;{ }^{* *} p<0.01$.

after the first one and for patient \#5 who presents the most severe muscle histopathology (Fig. 6A). CNBP mRNA expression appears to be similar in the two successive biopsies in all patients examined except for patient \#1 who presents a significant decrease of mRNA level in the second biopsy performed 10 years after the first one (Fig. 6B).

\section{Discussion}

The expansion of the CCTG repeat in the first intron of $C N B P$ gene results in a spliceopathy of downstream effector genes which is thought to account, at least in part, for multiorgan involvement $[7,8]$. At clinical level it has been observed that in DM2 as well as in DM1 patients, symptoms such as muscle weakness and myotonia undergo progressive worsening with increasing age [27]. Moreover, alternative splicing changes in skeletal muscle has been correlated with muscle weakness to some extent both in DM1 and DM2 patients [28]. We therefore studied the progression of DM2 through the analysis of histological and biomolecular disease markers in muscle biopsy. Our analysis of two successive biopsies of the same patient indicates a clear worsening of muscle histopathology over time and this worsening mostly occurs in type 2 fibers. Muscle degeneration appears to be more pronounced after a 10-year interval between the two successive biopsies; however it is already evident 

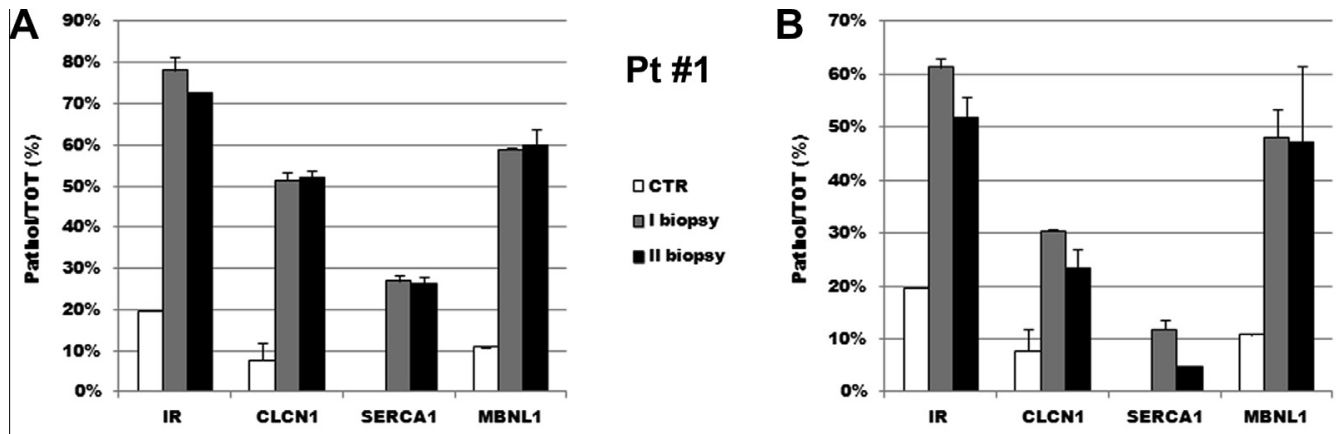

Pt \#2
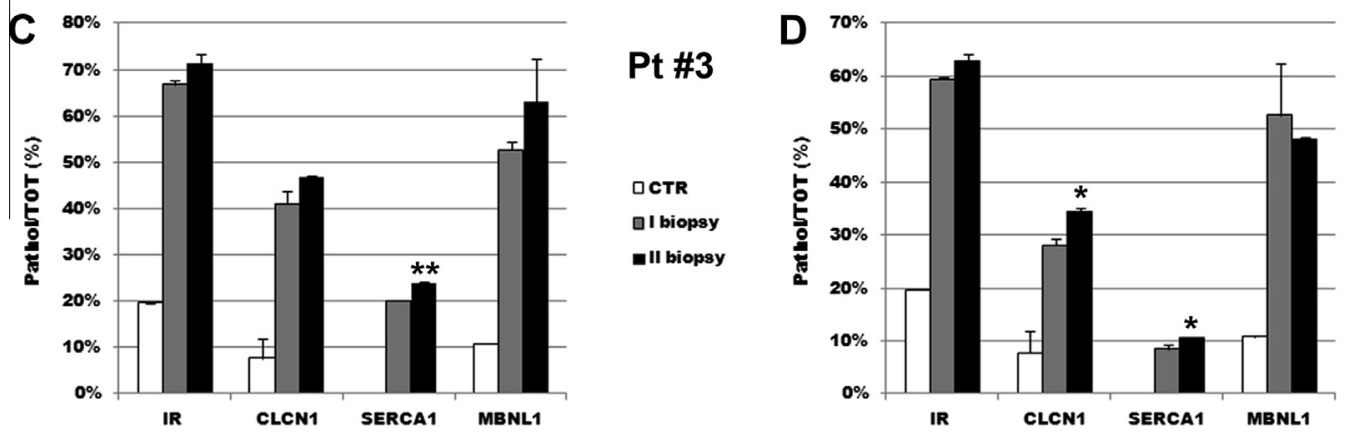

Pt \#4

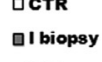

all biopsy

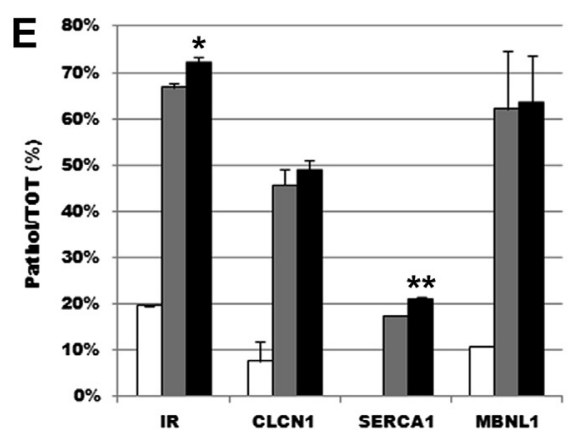

Pt \#5

QCTR

a biopsy

all biopsy

Fig. 5. Splicing analysis of the $I R, C L C N 1, S E R C A 1$ and $M B N L 1$ genes in muscle samples from 2 control subjects and from two successive biopsies of 5 DM2 patients (A-E). Histograms represent mean \pm SD. Student $t$-test: ${ }^{*} p<0.05 ;{ }^{* *} p<0.01$.

after 2 years. This degenerative process may explain the worsening of muscle symptoms like weakness and wasting and suggests that muscle biopsy represents a good tool to follow the progression of the disease. It has been reported that the expansion of DNA repeats increased by approximately $2 \mathrm{~kb}$ in the leukocytes during the 3-year interval between two successive blood donations $[4,29,30]$. In this study, because of the low amount of genomic DNA extracted from muscle tissue, we detected the DM2 mutation by a qualitative longPCR assay, which does not allow the estimation of the expansion size. However, our evaluation of dimensions of ribonuclear inclusions and MBNL1 foci demonstrates that both foci types increase in size over time in DM2 myonuclei indicating that CCUG repeats expand in skeletal muscle as well as in blood with increasing age and that a longer CCUG repeat is associated with sequestration of a larger amount of MBNL1, as previously reported for CUG repeat [31]. Thus the worsening of muscle symptoms with aging in DM2 patients may be caused, at the cellular level, by the progressive enlargement of repeats and by the consequent increase of the sequestration of factors involved in RNA processing and export to the cytoplasm. However, DM2 ribonuclear inclusions appear to be larger than DM1 and sequester a larger amount of MBNL1. Therefore, the depletion of MBNL1 from nucleoplasm seems to be more extensive in DM2 than in DM1 despite DM1 is considered a more severe disease. It appears likely that in DM1an additional pathogenic mechanism not shared by DM2 may exist, since sequestration of MBNL1 evidently has a central role in splicing misregulation in both types of DM. It has been reported that in DM1 a combined effect of decreased MBNL1 and increased CUGBP1 activity leads to misregulated alternative splicing and other changes of the muscle transcriptome [25,32], however evidence that CUGBP1 upregulation also occurs in DM2 is conflicting [31-33]. Our data indicate that CUGBP1 protein is not overexpressed in DM2 muscle biopsies as compared to controls as previously reported 


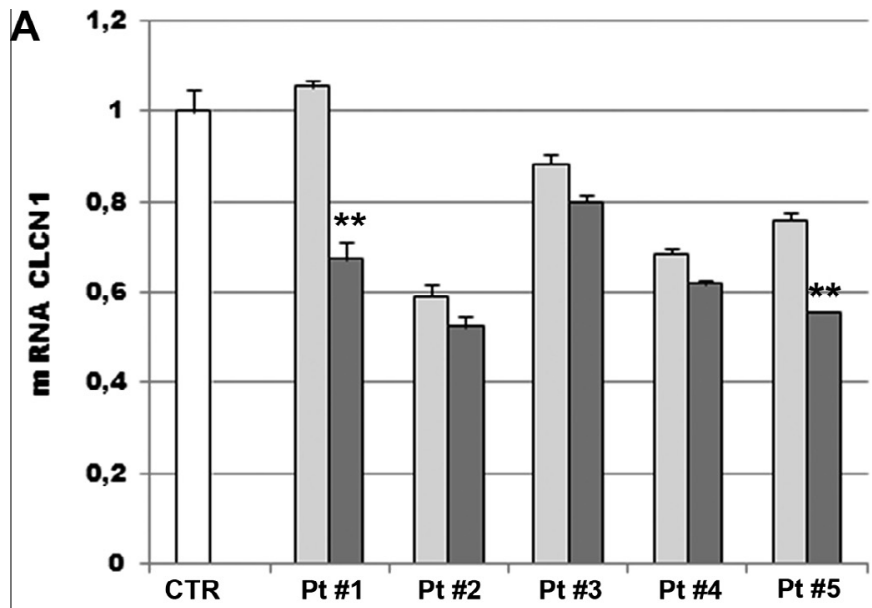

\section{I biopsy \\ 口 II biopsy}

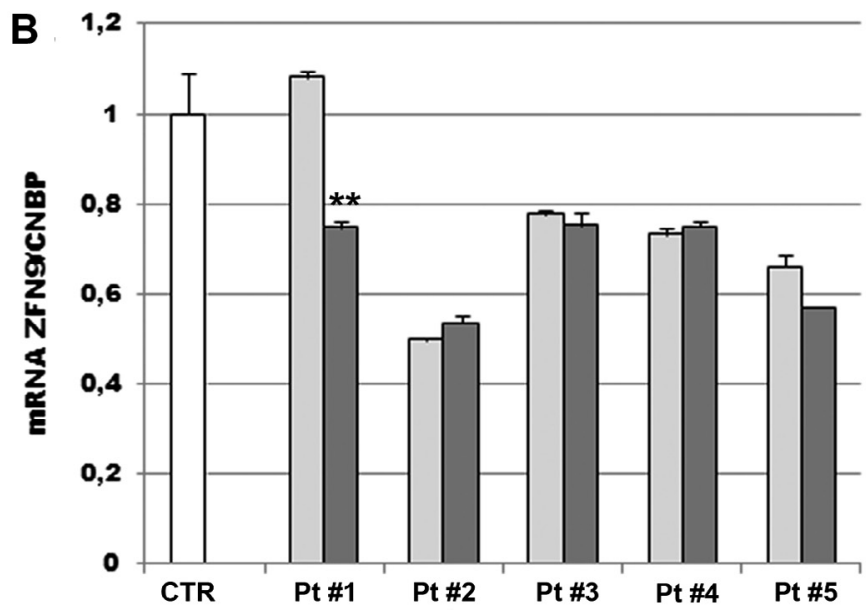

QI biopsy

all biopsy

Fig. 6. Results of QRT-PCR experiments to quantify the expression level of the $C L C N 1$ (A) and ZNF9/CNBP (B) mRNA in muscle samples from 2 control subjects and from two successive biopsies of $5 \mathrm{DM} 2$ patients. Each experiment has been performed in triplicate and the relative amount of the $C L C N 1$ transcripts has been determined using the $\beta_{2}$-microglobulin as endogenous control gene. Histograms represent mean \pm SD. Student $t$-test: ${ }^{* *} p<0.001$.

by other Authors [33,34]. Furthermore, it is noteworthy that in patients examined in our work, CUGBP1 expression level increases during aging. It has been reported that aging increases the amounts of CUGBP1 in the liver and adipose tissue $[35,36]$ through the formation of a multi-protein complexes of CUGBP1 such as CUGBP1-eIF2 [37]. Interestingly, formation of an identical complex has been observed in skeletal muscle of patients with DM2 in which this complex up-regulates translation of proteins involved in muscle development [32]. Also, a reduction of CNBP levels may play a role in DM2 pathology and might explain phenotypic differences between DM1 and DM2 [34,38-40]. In this work we have observed a reduction of CNBP both at the mRNA and protein level in DM2 muscle, however this reduction is not related to a worsening of muscle histology.

Since DM2 multisystemic phenotype is associated to a spliceopathy of several genes $[7,8]$, we have analyzed if the worsening of clinical symptoms may be related to an increase of the expression of the pathological isoforms of some genes involved in DM2 pathology. However, the analysis of alternative splicing of IR, SERCA1,
CLCN1and MBNL1 genes in two successive muscle biopsies does not evidence a worsening of alternative splicing alterations even after a 10-year interval between the two biopsies. Myotonia is the primary symptom in people who have DM and it gets worse over time [27]. It is well known that myotonia in DM patients is caused by the loss of CLCN1 mRNA and protein expression in DM1 skeletal muscle tissue due to aberrant splicing of the ClCN1 pre-mRNA [41,42]. In patients considered in this work we have not observed a worsening of CLCN1 splicing alteration though a decrease of CLCN1 mRNA has been observed in all patients. However we cannot assert if myotonia worsens over time in our DM2 cohort due to mexiletine treatment.

Taken together our data confirm that disease progression in DM2 is slow since histological and biomolecular alterations observed in skeletal muscle are minimal even after a 10-year interval. Moreover data indicate that muscle morphological alterations evolve more rapidly over time than the molecular changes thus indicating that muscle biopsy is a more sensitive tool than biomolecular markers to assess disease progression 
at muscle level. These observations would need to be confirmed with a larger sample of muscle biopsies.

Understanding the molecular mechanisms that play a role in DM disease progression is important for identifying new therapeutic agents, when considering that in DM patients not only skeletal muscle is affected, but also the central nervous system and the heart.

\section{Acknowledgements}

The Authors wish to thank Dr. Nadia Santo of Interdepartmental Centre of Advanced Microscopy (CIMA), University of Milan, for her technical support at confocal microscopy. This work was supported by unrestricted grant by FMM-Fondazione Malattie Miotoniche.

\section{References}

[1] Thornton CA, Griggs RC, Moxley RT. Myotonic dystrophy with no trinucleotide repeat expansion. Annal Neurol 1994;35:269-72.

[2] Ricker K, Koch MC, Lehmann-Horn F, et al. Proximal myotonic myopathy: a new dominant disorder with myotonia, muscle weakness, and cataracts. Neurology 1994;44:1448-52.

[3] Udd B, Krahe R, Wallgren-Pettersson C, et al. Proximal myotonic dystrophy: a family with autosomal dominant muscular dystrophy, cataracts, hearing loss and hypogonadism: heterogeneity of proximal myotonic syndromes? Neuromuscul Disord 1997;4:217-88.

[4] Liquori CL, Ricker K, Moseley ML, et al. Myotonic dystrophy type 2 caused by a CCTG expansion in intron 1 of ZNF9. Science 2001;293: 864-7.

[5] Brook JD, McCurrach ME, Harley HG, et al. Molecular basis of myotonic dystrophy: expansion of a trinucleotide (CTG) repeat at the $3^{\prime}$ end of a transcript encoding a protein kinase family member. Cell 1992;269:799-808.

[6] Mahadevan M, Tsilfidis C, Sabourin L, et al. Myotonic dystrophy mutation: an unstable CTG repeat in the $3^{\prime}$ untranslated region of the gene. Science 1992;255:1253-5.

[7] Timchenko NA, Cai ZJ, Welm AL, et al. RNA CUG repeats sequester CUGBP1 and alter protein levels and activity of CUGBP1. J Biol Chem 2001;276:7820-6.

[8] Fardaei M, Rogers MT, Thorpe HM, et al. Three proteins, MBNL, MBLL and MBXL, co-localize in vivo with nuclear foci of expanded-repeat transcripts in DM1 and DM2 cells. Hum Mol Genet 2002;11:805-14.

[9] Mankodi A, Teng-Umnuay P, Krym M, et al. Ribonuclear inclusions in skeletal muscle in myotonic dystrophy types 1 and 2. Ann Neurol 2003;54:760-8.

[10] Lin X, Miller JW, Mankodi A, et al. Failure of MBNL1-dependent postnatal splicing transitions in myotonic dystrophy. Hum Mol Genet 2006;15:2087-97.

[11] Osborne RJ, Thornton CA. RNA-dominant diseases. Hum Mol Genet 2006;15:R162-9.

[12] Vihola A, Bassez G, Meola G, et al. Histopathological differences of myotonic dystrophy type 1 (DM1) and PROMM/DM2. Neurology 2003;60:1854-7.

[13] Bassez G, Chapoy E, Bastuji-Garin S, et al. Type 2 myotonic dystrophy can be predicted by the combination of type 2 muscle fiber central nucleation and scattered atrophy. J Neuropathol Exp Neurol 2008;67:319-25

[14] Cardani R, Mancinelli E, Sansone V, et al. Biomolecular identification of (CCTG)n mutation in myotonic dystrophy type 2 (DM2) by FISH on muscle biopsy. Eur J Histochem 2004;48:437-42.
[15] Sallinen R, Vihola A, Bachinski LL, et al. New methods for molecular diagnosis and demonstration of the (CCTG)n mutation in myotonic dystrophy type 2 (DM2). Neuromuscul Disord 2004; $14: 274-83$.

[16] Bonifazi E, Vallo L, Giardina E, et al. A long PCR-based molecular protocol for detecting normal and expanded ZNF9 alleles in myotonic dystrophy type 2. Diagn Mol Pathol 2004;13:164-6.

[17] Sansone VA, Brigonzi E, Schoser B, et al. The frequency and severity of cardiac involvement in myotonic dystrophy type 2 (DM2): longterm outcomes. Int J Cardiol 2013;168:1147-53.

[18] Minnerop M, Weber B, Schoene-Bake JC, et al. The brain in myotonic dystrophy 1 and 2: evidence for a predominant white matter disease. Brain 2011;134:3530-46.

[19] Moxley 3rd RT, Meola G, Udd B, et al. Report of the 84th ENMC workshop: PROMM (proximal myotonic myopathy) and other myotonic dystrophy-like syndromes: 2nd workshop. 13-15th October 2000, Loosdrecht: The Netherlands. Neuromuscul Disord 2002;12:306-17.

[20] Miller SA, Dykes DD, Polesky HF. A simple salting out procedure for extracting DNA from human nucleated cells. Nucleic Acids Res 1988; $16: 1215$

[21] Dubowitz V. Muscle biopsy. In: Dubowitz V, editor. A Practical Approach. London: Bailliere Tindall; 1985. p. 19-40.

[22] Massa R, Panico MB, Caldarola S, et al. The myotonic dystrophy type 2 (DM2) gene product zinc finger protein 9 (ZNF9) is associated with sarcomeres and normally localized in DM2 patients' muscles. Neuropathol Appl Neurobiol 2010;36:275-84.

[23] Kimura T, Nakamori M, Lueck JD, et al. Altered mRNA splicing of the skeletal muscle ryanodine receptor and sarcoplasmic/endoplasmic reticulum $\mathrm{Ca}^{2+}$-ATPase in myotonic dystrophy type 1. Hum Mol Genet 2005;14:2189-200.

[24] Botta A, Rinaldi F, Catalli C, et al. The CTG repeat expansion size correlates with the splicing defects observed in muscles from myotonic dystrophy type 1 patients. J Med Genet 2008;45:639-46.

[25] Orengo JP, Chambon P, Metzger D, et al. Expanded CTG repeats within the DMPK $3^{\prime}$ UTR causes severe skeletal muscle wasting in an inducible mouse model for myotonic dystrophy. Proc Natl Acad Sci 2008; 105:2646-51.

[26] Botta A, Vallo L, Rinaldi F, et al. Gene expression analysis in myotonic dystrophy: indications for a common molecular pathogenic pathway in DM1 and DM2. Gene Expr 2007;13:339-51.

[27] Meola G, Sansone V, Marinou K, et al. Proximal myotonic myopathy: a syndrome with a favourable prognosis? J Neurol Sci 2002;193:89-96.

[28] Nakamori M, Sobczak K, Puwanant A, et al. Splicing biomarkers of disease severity in myotonic dystrophy. Ann Neurol 2013;74:862-72.

[29] Bachinski LL, Udd B, Meola G, et al. Confirmation of the type 2 myotonic dystrophy (CCTG)n expansion mutation in patients with proximal myotonic myopathy/proximal myotonic dystrophy of different European origins: a single shared haplotype indicates an ancestral founder effect. Am J Hum Genet 2003;73:835-48.

[30] Day JW, Ricker K, Jacobsen JF, et al. Myotonic dystrophy type 2: molecular, diagnostic and clinical spectrum. Neurology 2003;60: $657-64$.

[31] Miller JW, Urbinati CR, Teng-Umnuay P, et al. Recruitment of human muscleblind proteins to $(\mathrm{CUG})(\mathrm{n})$ expansions associated with myotonic dystrophy. EMBO J 2000;19:4439-48.

[32] Salisbury E, Schoser B, Schneider-Gold C, et al. Expression of RNA CCUG repeats dysregulates translation and degradation of proteins in myotonic dystrophy 2 patients. Am J Pathol 2009;175:748-62.

[33] Lin X, Miller JW, Mankodi A, et al. Failure of MBNL1-dependent post-natal splicing transitions in myotonic dystrophy. Hum Mol Genet 2006;15:2087-97.

[34] Pelletier R, Hamel F, Beaulieu D, et al. Absence of a differentiation defect in muscle satellite cells from DM2 patients. Neurobiol Dis 2009;36:181-90. 
[35] Wang GL, Salisbury E, Shi X, et al. HDAC1 cooperates with C/ EBP $\beta$ in the inhibition of liver proliferation in old mice. J Biol Chem 2008;283:26178-96.

[36] Karagiannides I, Thomou T, Tchkonia T, et al. Increased CUG triplet repeat-binding protein-1 predisposes to impaired adipogenesis with aging. J Biol Chem 2006;281:23025-33.

[37] Timchenko LT, Salisbury E, Wang GL, et al. Age-specific CUGBP1eIF2 complex increases translation of C/EBP $\beta$ in old liver. J Biol Chem 2006;281:32806-19.

[38] Huichalaf C, Schoser B, Schneider-Gold C, et al. Reduction of the rate of protein translation in patients with myotonic dystrophy 2. J Neurosci 2009;29:9042-9.

[39] Raheem O, Olufemi SE, Bachinski LL, et al. Mutant (CCTG)n expansion causes abnormal expression of zinc finger protein 9 (ZNF9) in myotonic dystrophy type 2. Am J Pathol 2010;177:3025-36.
[40] Chen W, Wang Y, Abe Y, et al. Haploinsufficiency for Znf9 in $\mathrm{Znf9}^{-} /^{-}$mice is associated with multiorgan abnormalities resembling myotonic dystrophy. J Mol Biol 2007;368:8-17.

[41] Charlet-B N, Savkur RS, Singh G, et al. Loss of the muscle-specific chloride channel in type 1 myotonic dystrophy due to misregulated alternative splicing. Mol Cell 2002;10:45-53.

[42] Mankodi A, Takahashi MP, Jiang H, et al. Expanded CUG repeats trigger aberrant splicing of $\mathrm{ClC}-1$ chloride channel pre-mRNA and hyperexcitability of skeletal muscle in myotonic dystrophy. Mol Cell 2002;10:35-44. 